\title{
Heating Two-Layer System with Pulsed Laser
}

\author{
M.K. El-Adawi ${ }^{1}$, H.M.Al-Theeb ${ }^{2}$ \\ ${ }^{1}$ Physics Department - Faculty of Education, Ain Shams University - Helliopolis - Cairo, Egypt \\ ${ }^{2}$ Physics Department - Faculty of Science for Girls- Dammam University, Dammam, P.O.Box838, Pincode31113, Saudi Arabia.
}

\begin{abstract}
Laser heating of two layer system is studied. The front layer is a homogeneous thin film having constant optical surface absorptance " $A_{f}$ ". This layer is of finite thickness. It is deposited on a thick substrate. The temperature profiles within the front layer and the substrate are obtained. Laplace integral transform technique is applied to solve the related heat diffusion equation of parabolic type. Computations for silicon-glass two layer system are given as an illustrative example.
\end{abstract}

Keywords: Pulsed laser heating, Laplace integral transform, heating Silicon-Glass two layer systems, constant optical absorptance, laser pulse parameters effect.

\section{Introduction}

Laser-solid interaction is a serious problem that has aroused the interest of many investigators [1-28]. It has different industrial applications, especially in the fields of material processing, such as spot welding, scribing, drilling of holes, laser cutting and laser glazing [1-5].

Pulsed lasers have been successfully applied for annealing of radiation damage associated with ion implantation in "Si" during device fabrication [9].

The pulse shape is important in determining the resulting thermal response of the target. In the semiconductor industry laser is used for local diffusion and alloying to form p-n junctions. Different theoretical models are made to study the laser heating problem.

Laser pulses of moderate power are used in a variety of fields, for example, to alter thin films on low-conductivity substrates especially for information recording [2-6].

The laser source function is assumed either constant (CW) laser or pulsed laser.

In the present trial, laser thermal response of a two-layer system is studied. The two-layer system is composed of a thin film of finite thickness "d" is deposited on a thick substrate.

Both layers are in perfect thermal contact. The system is subjected to laser source. The pulse shape is published elsewhere [25]. Laplace integral transform is applied to solve the heating problem and to get the thermal fields within the film layer and within the substrate. These functions are obtained in terms of the laser pulse parameters.

This makes it possible to determine the thermal penetration depth in the target and to determine the time required to initiate melting in the front layer. This in turn is important in studying the dynamics of pulsed laser interaction.
Computations on Silicon-Glass two-layer system is made and is given as an illustrative example.

\section{Mathematical Formulation of the Problem:}

In setting up the problem, it is assumed that laser $\mathrm{q}_{\mathrm{o}}(\mathrm{t})$ is incident on the front surface of the system in a normal direction (Fig. 1). The received power is partly absorbed and partly reflected. The absorbed part is $\left(A_{f} \mathrm{q}_{0}(\mathrm{t})\right)$ where $\mathrm{A}_{\mathrm{f}}$ is the absorptance of the thin film front surface. The thickness of the thin film is "d" microns. Two coincident axes $\mathrm{x}$ and $\mathrm{z}=(\mathrm{x}-\mathrm{d})$, along the direction of the incident laser beam are considered.

The boundary $z=0$ represents the interface between the two layers [19]. Thermal losses arising from any mode other than convection are neglected. The physical parameters together with the optical absorptance " $\mathrm{A}_{\mathrm{f}}$ " are assumed to be temperature independent.

The heat flow is considered one-dimensional [1, 19], Laplace integral transform technique is used to obtain the required solutions.

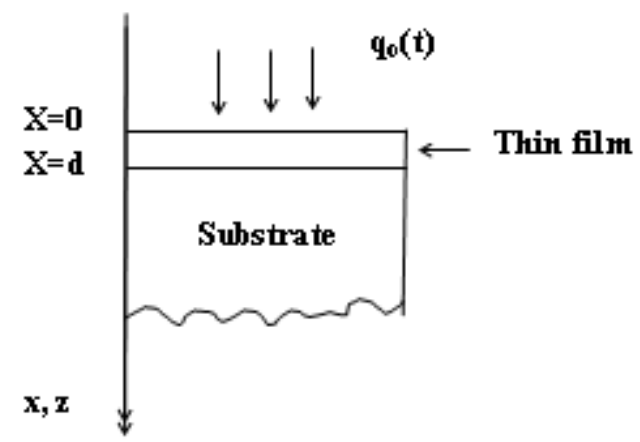

Figure 1: Schematic view of the considered model.

The heat diffusion equation in the film layer can be written in the form:

$$
\frac{\partial \theta_{f}(x, t)}{\partial x}=\alpha_{f} \frac{\partial^{2} \theta_{f}(x, t)}{\partial x^{2}}, \quad \begin{gathered}
t_{m}>t>0, \\
0 \leq x \leq d
\end{gathered}
$$




\section{International Journal of Science and Research (IJSR) \\ ISSN (Online): 2319-7064 \\ Index Copernicus Value (2013): 6.14 | Impact Factor (2014): 5.611}

Where, $\alpha=\lambda / \rho c_{p}$, is the thermal diffusivity $\mathrm{m}^{2} / \mathrm{s}, \mathrm{t}_{\mathrm{m}}$, is the time required to initiate melting, $\theta=\left(\mathrm{T}-\mathrm{T}_{\mathrm{o}}\right)$ is the excess temperature relative to the ambient temperature $T_{0}$.

For the substrate layer.

$$
\frac{\partial \theta_{\rho}(z, t)}{\partial t}=\alpha_{\rho} \frac{\partial^{2} \theta_{\rho}(z, t)}{\partial z^{2}}, \quad o \leq z \leq \infty, t<t_{m}
$$

$\lambda, \mathrm{W} / \mathrm{m} . \mathrm{K}$ is the thermal conductivity,

$\rho, \mathrm{kg} / \mathrm{m}^{3}$ is the density,

$\mathrm{C}_{\mathrm{p}},(\mathrm{J} / \mathrm{kg} . \mathrm{K})$ is the specific heat.

The system of equation 1 and 2 is subjected to the following initial and boundary conditions:
i) $\quad \theta_{\mathrm{f}}(\mathrm{x}, \mathrm{o})=0$
ii) $\quad \theta_{\rho}(\mathrm{z}, \mathrm{o})=0$
iii ) $\quad \theta_{\rho}(\infty, \mathrm{t})=0$

The condition at the front surface $\mathrm{x}=0$

$$
\left.\lambda_{f} \frac{\partial \theta_{f}(x, t)}{\partial x}\right|_{x=0}=A_{f}(T) q_{o}(t)-h_{o} \theta_{f}(o, t)
$$

Where $" h_{0}$ " is the heat transfer coefficient at the front surface of the system.

The source laser function is suggested by the author elsewhere [25] in the form:

$$
q(t)=q_{\max }\left(\frac{t_{d}-t}{t_{d}-t_{o}}\right)^{m}\left(\frac{t}{t_{o}}\right), m=\left(\frac{t_{d}}{t_{o}}-1\right)
$$

Where $\mathrm{q}_{\max }$, is the maximum power density $\mathrm{W} / \mathrm{m} 2$ of the pulse.

$t_{d}$ is the time duration of the pulse,

$\mathrm{t}_{\mathrm{o}}$ is the time interval after which the power density reaches its maximum value $\mathrm{q}_{\max }$.

The condition of continuity of the interface $x=d$,

$$
\begin{array}{r}
\theta_{\mathrm{f}}(\mathrm{d}, \mathrm{t})=\theta_{\rho}(\mathrm{z}=\mathrm{o}, \mathrm{t}) \\
-\left.\lambda_{f} \frac{\partial \theta_{f}(x, t)}{\partial x}\right|_{x=d}=-\left.\lambda_{\rho} \frac{\partial \theta_{\rho}(z, t)}{\partial z}\right|_{z=(x-d)=0}
\end{array}
$$

Together with the integrated heat balance equation:

$$
\begin{aligned}
\int_{0}^{\mathrm{t}} \mathrm{A}_{\mathrm{f}} \mathrm{q}(\mathrm{t}) \mathrm{dt}= & \int_{\mathrm{o}}^{\mathrm{d}} \lambda \underset{\mathrm{f}}{\lambda} \mathrm{C}_{\mathrm{p}_{\mathrm{f}}} \theta_{\mathrm{f}}(\mathrm{x}, \mathrm{t}) \mathrm{dx}+\int_{\mathrm{o} \rho}^{\infty} \rho \mathrm{C}_{\mathrm{p}_{\rho}} \theta_{\rho}(\mathrm{z}, \mathrm{t}) \mathrm{dz}+ \\
& \int_{0}^{\mathrm{t}} \mathrm{h}_{\mathrm{o}} \theta_{\mathrm{f}}(\mathrm{o}, \mathrm{t}) \mathrm{dt}
\end{aligned}
$$

Taking Laplace transform with respect to the time variable for both equations (1) and (2) one gets:

$$
s \bar{\theta}_{f}(x, s)-\theta_{f}(x, o)=\alpha_{f} \frac{\partial^{2} \theta_{f}(x, s)}{\partial x^{2}}=o
$$

and

$$
s \bar{\theta}_{\rho}(z, s)-\theta_{\rho}(z, o)=\alpha_{\rho} \frac{\partial^{2} \theta_{\rho}(z, s)}{\partial z^{2}}
$$

Where $\bar{\theta}$ is the Laplace transform of $\theta, \mathrm{s}$ is the variable in Laplace domain.

One has also to transform the boundary and initial conditions to Laplace domain, this gives:

$$
\begin{array}{ll}
\text { a) } & \bar{\theta}_{\mathrm{f}}(\mathrm{x}, \mathrm{o})=0 \\
\text { b) } & \bar{\theta}_{\rho}(\mathrm{z}, \mathrm{o})=0 \\
\text { c) } & \bar{\theta}_{\rho}(\infty, \mathrm{s})=0 \\
\text { d) } & \bar{\theta}_{\rho}(\infty, \mathrm{o})=0
\end{array}
$$

Equation (2-4) is transformed in the form:

$$
-\left.\lambda_{f} \frac{\partial \bar{\theta}_{f}(x, s)}{\partial x}\right|_{x=0}=A_{f} G(s)-h_{o} \bar{\theta}_{f}(o, s)
$$

Where $\overline{\mathrm{G}}(\mathrm{s})$ is the Laplace transform of the source function $\mathrm{q}_{\mathrm{o}}(\mathrm{t})$,

Equation (6) is transformed in the form:

$$
\bar{\theta}_{\mathrm{f}}(\mathrm{d}, \mathrm{s})=\bar{\theta}_{\rho}(\mathrm{o}, \mathrm{s})
$$

Equation (7) is transformed as:

$$
-\left.\lambda_{f} \frac{\partial \bar{\theta}_{f}(x, s)}{\partial x}\right|_{x=d}=-\left.\lambda_{\rho} \frac{\partial \bar{\theta}_{\rho}(z, s)}{\partial z}\right|_{z=0}
$$

The heat balance equation (2-8) is transformed in the form:

$$
\frac{A_{f} G(s)}{s}=\int_{0}^{d} \rho_{f} C_{p_{f}} \bar{\theta}_{f}(x, s) d x+\int_{0}^{\infty} \rho_{s} C_{p_{s}} \bar{\theta}_{s}(z, s) d z+\frac{h_{o} \bar{\theta}_{f}(0, s)}{s}
$$

Considering equation (11), one can write equations (9) and (10) in the form:

$$
s \bar{\theta}_{f}(x, s)-\alpha_{f} \frac{\partial^{2} \bar{\theta}_{f}(x, s)}{\partial x^{2}}=0
$$

and

$$
s \bar{\theta}_{\rho}(z, s)-\alpha_{\rho} \frac{\partial^{2} \bar{\theta}_{p}(z, s)}{\partial z^{2}}=0
$$

The solution of both equations (16) and (17) can be written in the form: 
$\bar{\theta}_{f}(x, s)=C_{1} \exp \left(-\sqrt{\frac{s}{\alpha_{f}}} x\right)+C_{2} \exp \left(-\sqrt{\frac{s}{\alpha_{f}}} x\right)$

and

$$
\bar{\theta}_{\rho}(z, s)=C_{3} \exp \left(-\sqrt{\frac{s}{\alpha_{\rho}}} z\right)+C_{4} \exp \left(+\sqrt{\frac{s}{\alpha_{\rho}}} z\right)
$$

To get finite solution at $\mathrm{z}=\infty$, one must put $\mathrm{C}_{4}=0$.

Thus, the solution can be rewritten in the form:

$$
\bar{\theta}_{\rho}(z, s)=C_{3} \exp \left(-\sqrt{\frac{s}{\alpha_{\rho}}} z\right)
$$

Substituting solutions (18) and (20) into equations (12), (13), (14) and (15) one gets the following system of equations:

$$
\begin{array}{r}
\left(h_{o}-\lambda_{f} \sqrt{\frac{s}{\alpha_{f}}}\right) C_{1}+\left(h_{o}+\lambda_{f} \sqrt{\frac{s}{\alpha_{f}}}\right) C_{2}=A_{f} G(s)(21) \\
\exp \left(\sqrt{\frac{\mathrm{s}}{\alpha_{\mathrm{f}}} \mathrm{d}}\right) \mathrm{C}_{1}+\exp \left(-\sqrt{\frac{\mathrm{s}}{\alpha_{\mathrm{f}}} \mathrm{d}}\right) \mathrm{C}_{2}-\mathrm{C}_{3}=0
\end{array}
$$

$$
-\lambda_{f} \sqrt{\frac{s}{\alpha_{f}}} \exp \left(\sqrt{\frac{s}{\alpha_{f}}} d\right) C_{1}+\lambda_{f} \sqrt{\frac{s}{\alpha_{f}}} \exp \left(\sqrt{\frac{s}{\alpha_{f}}} d\right) C_{2}-\lambda p \sqrt{\frac{s}{\alpha_{p}}} C_{3}=0
$$

$$
\begin{aligned}
& \left\lfloor\rho_{f} C_{p_{f}} \sqrt{\frac{\alpha_{f}}{s}} \exp \left(\sqrt{\frac{s}{\alpha_{f}}} d\right)-\rho_{f} C_{p_{f}} \sqrt{\frac{\alpha_{f}}{s}}+\frac{h_{o}}{s}\right] C_{1}+ \\
& {\left[-\rho_{f} C_{p_{f}} \sqrt{\frac{\alpha_{f}}{s}} \exp \left(-\sqrt{\frac{s}{\alpha_{f}}} d\right)+\rho_{f} C_{p_{f}} \sqrt{\frac{\alpha_{f}}{s}}+\frac{h_{o}}{s}\right] C_{2}+\rho_{\rho} C_{p_{\rho}} \sqrt{\frac{\alpha_{\rho}}{s}} C_{3}=\frac{A_{f} G(s)}{s}}
\end{aligned}
$$

Solving this system of equations for $\mathrm{C}_{1}, \mathrm{C}_{2}$ and $\mathrm{C}_{3}$ one gets:

$$
\begin{aligned}
& C_{1}=A_{f} G(s) \frac{\lambda_{f} \sqrt{\frac{s}{\alpha_{f}}} \exp \left(-\sqrt{\frac{s}{\alpha_{f}}} d\right)-\lambda_{s} \sqrt{\frac{s}{\alpha_{s}}} \exp \left(-\sqrt{\frac{s}{\alpha_{f}}} d\right)}{\left(\lambda_{s} \sqrt{\frac{s}{\alpha_{s}}} h_{o}+\lambda_{f}^{2} \frac{s}{\alpha_{f}}\right) \sinh \left(\sqrt{\frac{s}{\alpha_{f}}} d\right)+\left(\lambda_{p} \lambda_{f} \sqrt{\frac{s}{\alpha_{f}}} \sqrt{\frac{s}{\alpha_{s}}}+h_{o} \lambda_{f} \sqrt{\frac{s}{\alpha_{f}}}\right) \operatorname{Cosh}\left(\sqrt{\frac{s}{\alpha_{f}}} d\right)} \\
& C_{2}=A_{f} G(s) \frac{\lambda_{f} \sqrt{\frac{s}{\alpha_{f}}} \exp \left(-\sqrt{\frac{s}{\alpha_{f}}} d\right)+\lambda p \sqrt{\frac{s}{\alpha_{s}}} \exp \left(\sqrt{\frac{s}{\alpha_{f}}} d\right)}{\left(\lambda_{s} \sqrt{\frac{s}{\alpha_{s}}} h_{o}+\lambda_{f}^{2} \frac{s}{\alpha_{f}}\right) \sinh \left(\sqrt{\frac{s}{\alpha_{f}}} d\right)+\left(\lambda_{p} \lambda_{f} \sqrt{\frac{s}{\alpha_{f}}} \sqrt{\frac{s}{\alpha_{p}}}+h_{o} \lambda_{f} \sqrt{\frac{s}{\alpha_{f}}}\right) \operatorname{Cosh}\left(\sqrt{\frac{s}{\alpha_{f}}} d\right)} \\
& C_{3}=A_{f} G(s) \frac{2 \lambda_{f} \sqrt{\frac{s}{\alpha_{f}}}}{\left(\lambda_{p} \sqrt{\frac{s}{\alpha_{p}}} h_{o}+\lambda_{f}^{2} \frac{s}{\alpha_{f}}\right) \sinh \left(\sqrt{\frac{s}{\alpha_{f}}} d\right)+\left(\lambda p \lambda_{f} \sqrt{\frac{s}{\alpha_{f}}} \sqrt{\frac{s}{\alpha_{p}}}+h_{o} \lambda_{f} \sqrt{\frac{s}{\alpha_{f}}}\right) \operatorname{Cosh}\left(\sqrt{\frac{s}{\alpha_{f}}} d\right)}
\end{aligned}
$$

Substituting for $\mathrm{C}_{1}, \mathrm{C}_{2}$ and $\mathrm{C}_{3}$ in the solutions expressed by equations (18) and (20), and rearranging the obtained terms one can finally write the solutions in the form: 


$$
\begin{aligned}
& \bar{\theta}_{f}(x, s)=A_{f} G(s) \mid\left\{\exp \left(-\sqrt{\frac{s}{\alpha_{f}}} x\right)+\exp \left(-\sqrt{\frac{s}{\alpha_{f}}}(2 d-x)\right\}+\frac{\lambda_{\rho}}{\lambda_{f}} \sqrt{\frac{\alpha_{f}}{\alpha_{\rho}}}\right. \\
& {\left[\exp \left(-\sqrt{\frac{s}{\alpha_{f}}} x\right)-\exp \left(-\sqrt{\frac{s}{\alpha_{f}}}(2 d-x)\right)\right\} \div\left[\left\{\frac{\lambda_{\rho} h_{o}}{\lambda_{f}} \sqrt{\frac{\alpha_{f}}{\alpha_{\rho}}}+\lambda_{\rho} \sqrt{\frac{s}{\alpha_{\rho}}}+\lambda_{f} \sqrt{\frac{s}{\alpha_{f}}}+h_{o}\right\}\right.} \\
& \left.\left\{1-\gamma \exp \left(-2 \sqrt{\frac{s}{\alpha_{f}}} d\right)\right\}\right]
\end{aligned}
$$

and

$$
\begin{aligned}
& \bar{\theta}_{s}(z, s)=A_{f} G(s)\left\{4 \exp \left(-\left(\frac{z}{\sqrt{\alpha_{p}}}+\frac{d}{\sqrt{\alpha_{f}}}\right) \sqrt{s}\right)\right\} \div\left\{\left(\frac{\lambda_{p} h_{o}}{\lambda_{f}} \sqrt{\frac{\alpha_{f}}{\alpha_{p}}}+\lambda_{p} \sqrt{\frac{s}{\alpha_{p}}}+\lambda_{f} \sqrt{\frac{s}{\alpha_{f}}}+h_{o}\right) .\right. \\
& \left.\left[1-\gamma \exp \left(-2 \sqrt{\frac{s}{\alpha_{f}}} d\right)\right]\right\}
\end{aligned}
$$

Where

$$
\gamma=\frac{\left(\frac{\lambda_{\rho} h_{o}}{\lambda_{f}} \sqrt{\frac{\alpha_{f}}{\alpha_{\rho}}}+\lambda_{f} \sqrt{\frac{s}{\alpha_{f}}}\right)-\left(\lambda_{\rho} \sqrt{\frac{s}{\alpha_{\rho}}}+h_{o}\right)}{\left(\frac{\lambda_{\rho} h_{o}}{\lambda_{f}} \sqrt{\frac{\alpha_{f}}{\alpha_{\rho}}}+\lambda_{f} \sqrt{\frac{s}{\alpha_{f}}}\right)+\left(\lambda_{\rho} \sqrt{\frac{s}{\alpha_{\rho}}}+h_{o}\right)}
$$

Equation (30) indicates that:

$$
0 \leq \gamma \leq 1
$$

Moreover, discussing the order of magnitudes of the different terms in the expression (eq. 30) for $\gamma$, one can conclude that :

$$
\begin{aligned}
& \text { Since } \frac{1}{1-a}=\sum_{n=o}^{\infty} a^{n},|a|<1 \quad[29] \text { (33) form: } \\
& \bar{\theta}_{\mathrm{f}}(\mathrm{x}, \mathrm{s})=\frac{\mathrm{A}_{\mathrm{f}} \mathrm{G}(\mathrm{s}) \sqrt{\alpha_{\mathrm{f}} \alpha_{\rho}}}{\lambda_{\mathrm{f}} \sqrt{\alpha_{\rho}}+\lambda_{\rho} \sqrt{\alpha_{\mathrm{f}}}}\left\{\left[\exp \left(-\sqrt{\frac{\mathrm{s}}{\alpha_{\mathrm{f}}}}\right)+\exp \left(-\sqrt{\frac{\mathrm{s}}{\alpha_{\mathrm{f}}}}(2 \mathrm{~d}-\mathrm{x})\right)\right]+\frac{\lambda_{\rho}}{\lambda_{\mathrm{f}}} \sqrt{\frac{\alpha_{\mathrm{f}}}{\alpha_{\rho}}}\right. \\
& \left.\left[\exp \left(-\sqrt{\frac{\mathrm{s}}{\alpha_{\mathrm{f}}}} \mathrm{x}\right)-\exp \left(-\sqrt{\frac{\mathrm{s}}{\alpha_{\mathrm{f}}}}(2 \mathrm{~d}-\mathrm{x})\right)\right]\right\} \div\left\{\left(\sqrt{\mathrm{s}}+\frac{\mathrm{h}_{\mathrm{o}} \sqrt{\alpha_{\mathrm{f}}}}{\lambda_{\mathrm{f}}}\right) \sum_{\mathrm{n}=0}^{\infty} \gamma^{\mathrm{n}} \exp \left(-2 \mathrm{n} \sqrt{\frac{\mathrm{s}}{\alpha_{\mathrm{f}}}} \mathrm{d}\right)\right.
\end{aligned}
$$

One can write

$$
\frac{1}{1-\gamma \exp \left(-2 \sqrt{\frac{s}{\alpha_{f}} d}\right)}=\sum_{n=o}^{\infty} \gamma^{n} \exp \left(-2 n \sqrt{\frac{s}{\alpha_{f}}} d\right)
$$

Substituting equations (34) into equations (28) and (29) one can write the solutions (28) and (29) in the following 


$$
\begin{gathered}
\bar{\theta}_{\rho}(z, s)=\frac{A_{f} G(s) \sqrt{\alpha_{f} \alpha_{p}}}{\lambda_{f} \sqrt{\alpha_{\rho}}+\lambda_{\rho} \sqrt{\alpha_{f}}}\left\{4 \exp \left(-\left(\sqrt{\frac{z}{\alpha_{\rho}}}+\frac{d}{\sqrt{\alpha_{f}}}\right) \sqrt{s}\right)\right\} \div \\
\left\{\left(\sqrt{s}+\frac{h_{o} \sqrt{\alpha_{f}}}{\lambda_{f}}\right) \sum_{n=0}^{\infty} \gamma^{n} \exp \left(-2 n \sqrt{\frac{s}{\alpha_{f}}} d\right)\right.
\end{gathered}
$$

For $\gamma=1$, then solutions (35) and (36) can be rewritten in the form:

$$
\begin{gathered}
\bar{\theta}_{f}(x, s)=\frac{A_{f} G(s) \sqrt{\alpha_{f} \alpha_{p}}}{\lambda_{f} \sqrt{\alpha_{\rho}}+\lambda_{\rho} \sqrt{\alpha_{f}}}\left\{\sum _ { n = 0 } ^ { \infty } \left[\exp \left(-\sqrt{\frac{s}{\alpha_{f}}}(2 n d+x)\right)+\exp \left(\sqrt{\frac{s}{\alpha_{f}}}((2 n+2) d-x)\right)+\right.\right. \\
\left.\frac{\lambda_{p}}{\lambda_{f}} \sqrt{\frac{\alpha_{f}}{\alpha_{\rho}}}\left(\exp \left(-\sqrt{\frac{s}{\alpha_{f}}}((2 n d+x))-\exp \sqrt{\frac{s}{\alpha_{f}}}((2 n+2) d-x)\right)\right]\right\} \div\left\{\left(\sqrt{s}+\frac{h_{o} \sqrt{\alpha_{f}}}{\lambda_{f}}\right)\right\} \\
\bar{\theta}_{\mathrm{s}}(\mathrm{z}, \mathrm{s})=\frac{\mathrm{A}_{\mathrm{f}} \mathrm{G}(\mathrm{s}) \sqrt{\alpha_{\mathrm{f}} \alpha_{\rho}}}{\lambda_{\mathrm{f}} \sqrt{\alpha_{\rho}}+\lambda_{\rho} \sqrt{\alpha_{\mathrm{f}}}} \frac{\sum_{\mathrm{n}=0}^{\infty} 4 \exp \left(-\left(\frac{2 \mathrm{nd}+\mathrm{d}}{\sqrt{\alpha_{\mathrm{f}}}}+\frac{\mathrm{z}}{\sqrt{\alpha_{\rho}}}\right) \sqrt{\mathrm{s}}\right)}{\sqrt{\mathrm{s}}+\frac{\mathrm{h}_{\mathrm{o}} \sqrt{\alpha_{\mathrm{f}}}}{\lambda_{\mathrm{f}}}}
\end{gathered}
$$

To get the inverse Laplace transform one has to apply the convolution theorem [29].

$$
L^{-1}\left\{f_{1}(s) f_{2}(s)\right\}=\int_{0}^{t} F_{1}(u) F_{2}(t-u) d u
$$

Using the standard tables [29-32] one gets the inverse Laplace transform of equations (37) and (38) in the form:

$$
\begin{aligned}
& \theta_{f}(x, t)=\frac{A_{f} q_{m}(s) \sqrt{\alpha_{f} \alpha_{\rho}}}{\left(\lambda_{f} \sqrt{\alpha_{\rho}}+\lambda_{\rho} \sqrt{\alpha_{f}}\right)\left(t_{d}-t_{0}\right)^{m} \mathrm{t}_{0}} \mid \int_{0}^{t}\left\{( t _ { d } - u ) ^ { m } u \sum _ { n = 0 } ^ { \infty } \left(\frac{\exp \left(-\frac{(2 n d+x)^{2}}{4 \alpha_{f} t}\right)}{\sqrt{\pi(t-u)}}-\frac{h_{o} \sqrt{\alpha_{f}}}{\lambda_{f}}\right.\right. \\
& \exp \left(\frac{h_{o}(2 n d+x)}{\lambda_{f}}\right) \exp \left(\frac{h_{o}^{2} \alpha_{f}}{\lambda_{f}^{2}}(t-u)\right) \operatorname{erfc}\left(\frac{h_{o} \sqrt{\alpha_{f}(t-u)}}{\lambda_{f}}+\frac{2 n d+x}{\sqrt{4 \alpha_{f}(t-u)}}\right)+ \\
& \frac{\exp \left(-\frac{(2 n+2) d-x)^{2}}{4 \alpha_{f}(t-u)}\right)}{\sqrt{\pi(t-u)}}-\left(\frac{h_{o} \sqrt{\alpha_{f}}}{\lambda_{f}}\right) \exp \left(\frac{h_{o}((2 n+2) d-x)}{\lambda_{f}}\right) \exp \left(\frac{h_{o}{ }^{2} \alpha_{f}}{\lambda_{f}^{2}}(t-u)\right) \\
& \left.\operatorname{erfc}\left(\frac{h_{o} \sqrt{\alpha_{f}(t-u)}}{\lambda_{f}^{2}}+\frac{(2 n+2) d-x}{\sqrt{4 \alpha_{f}(t-u)}}\right)\right)+\frac{\lambda_{\rho}}{\lambda_{f}} \sqrt{\frac{\alpha_{f}}{\alpha_{\rho}}} \\
& \sum_{n=0}^{\infty}\left(\frac{\exp \left(-\frac{(2 n d+x)^{2}}{4 \alpha_{f}(t-u)}\right)}{\sqrt{\pi(t-u)}}-\frac{h_{o} \sqrt{\alpha_{f}}}{\lambda_{f}} \exp \left(\frac{h_{o}(2 n d+x)}{\lambda_{f}}\right) \exp \left(\frac{h_{o}{ }^{2} \alpha_{f}}{\lambda_{f}^{2}} t\right)\right. \\
& \operatorname{erfc}\left(\frac{h_{o} \sqrt{\alpha_{f}(t-u)}}{\lambda_{f}}+\frac{2 n d+x}{\sqrt{4 \alpha_{f}(t-u)}}\right)-\frac{\exp \left(\frac{((2 n+2) d-x)^{2}}{4 \alpha_{f}(t-u)}\right)}{\sqrt{\pi(t-u)}}+\frac{h_{o} \sqrt{\alpha_{f}}}{\lambda_{f}} \\
& \exp \left(\frac{h_{o}((2 n+2) d-x)}{\lambda_{f}}\right) \exp \left(\frac{h_{o}{ }^{2} \alpha_{f}}{\lambda_{f}^{2}}(t-u)\right) \\
& \left.\left.\left.\operatorname{erfc}\left(\frac{h_{o} \sqrt{\alpha_{f}(t-u)}}{\lambda_{f}^{2}}+\frac{(2 n+2) d-x}{\sqrt{4 \alpha_{f}(t-u)}}\right)\right)\right\} d u\right]
\end{aligned}
$$




\section{International Journal of Science and Research (IJSR)

$$
\begin{aligned}
& \theta_{\rho}(z, t)=\frac{4 A_{f} q_{m} \sqrt{\alpha_{f} \alpha_{\rho}}}{\left(\lambda_{f} \sqrt{\alpha_{\rho}}+\lambda_{\rho} \sqrt{\alpha_{f}}\right)\left(t_{d}-t_{0}\right) m t_{0}} \int_{0}^{t}\left\{\left(t_{d}-u\right)^{m} u \sum_{n=0}^{\infty}\left(\frac{\exp \left(-\left(\frac{(2 n d+d)^{2}}{4 \alpha_{f}(t-u)}+\frac{z^{2}}{4 \alpha_{\rho}(t-u)}\right)\right)}{\sqrt{\pi(t-u)}}\right)\right. \\
& -\frac{h_{o} \sqrt{\alpha_{f}}}{\lambda_{f}} \exp \left(\frac{\left(\frac{h_{o}(2 n d+d)^{2}}{4 \alpha_{f}(t-u)}\right)+\frac{z^{2}}{4 \alpha_{\rho}(t-u)}}{\lambda_{f}}\right)-\exp \left(\frac{h_{o}{ }^{2} \alpha_{f}}{\lambda_{f}^{2}}(t-u)\right) \\
& \left.\left.\left.\operatorname{erfc}\left(\frac{h_{o} \sqrt{\alpha_{f} t}}{\lambda_{f}}+\frac{2 n d+d}{\sqrt{4 \alpha_{f}(t-u)}}+\frac{z}{4 \alpha_{\rho}(t-u)}\right)\right)\right\} d u\right]
\end{aligned}
$$

Equations (40) and (41) show the temperature profiles in the thin film layer and in the substrate of the chosen two layer target.

\section{Computations}

As illustrative example the temperature commuted for the following two-layer system

\section{i. Silicon-glass}

The physical properties of the considered materials are given in Table 1 [32-34]

Table 1: The physical and optical properties of the considered materials [32-34]

\begin{tabular}{|c|c|c|c|c|c|c|c|}
\hline Material & $\begin{array}{c}\mathbf{\rho} \\
\left(\mathbf{k g m}^{-\mathbf{3}}\right)\end{array}$ & $\begin{array}{c}\boldsymbol{\lambda} \\
\left(\mathbf{W m}^{-\mathbf{1}} \mathbf{K}^{-\mathbf{1}}\right)\end{array}$ & $\begin{array}{c}\boldsymbol{\alpha} \\
\left(\mathbf{m}^{2} \mathbf{s}^{-\mathbf{1}}\right)\end{array}$ & $\begin{array}{c}\mathbf{C}_{\mathbf{p}} \\
\left(\mathbf{J k g}^{-1} \mathbf{K}^{-\mathbf{1}}\right)\end{array}$ & $\mathbf{A}_{1}$ & $\begin{array}{c}\mathbf{A} 2 \\
\mathbf{K}^{-\mathbf{1}}\end{array}$ & $\mathbf{T m}, \mathbf{K}$ \\
\hline Silicon & $2.328 \times 10^{3}$ & $1.5 \times 10^{2}$ & $9.2 \times 10^{-5}$ & 700 & 0.678 & $3.12 \times 10^{-5}$ & 1688 \\
\hline Glass & 2707 & 0.75 & $0.035 \times 10^{-5}$ & $0.8 \times 10^{3}$ & - & - & - \\
\hline
\end{tabular}

The laser pulse is characterized by the following set of parameters:

$$
\begin{aligned}
& q_{\max }=1.25 \times 10^{9} \mathrm{~W} / \mathrm{m}^{2}, \\
& t_{d}=40 \mu \mathrm{s}, \text { to }=10 \mu \mathrm{s}, \text { and } m=3
\end{aligned}
$$

The heat transfer of the front surface

$$
\mathrm{h}_{\mathrm{o}}=1000 \mathrm{~W} / \mathrm{m}^{2} \mathrm{~K}
$$

The thickness of the thin film $=\mathrm{d}=10$ microns.

The function $\theta_{f}(0, t)$ and $\theta_{f}(x, t=34 \mu s)$, are computed for the system Silicon-glass. The obtained results are illustrated graphically in figures (1) and (2) respectively.

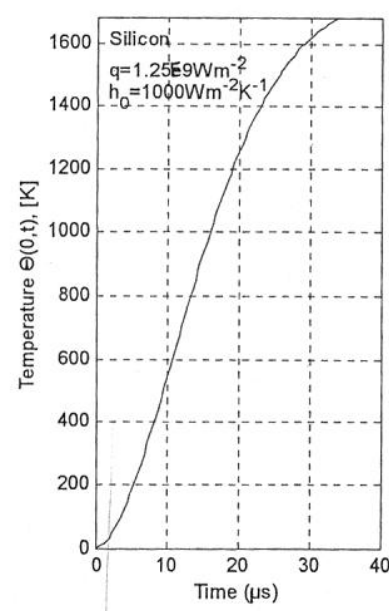

Fig. 2 : The variation of the temperature of the front surface as a function of the exposure times for the two-layer system (silicon-glass)

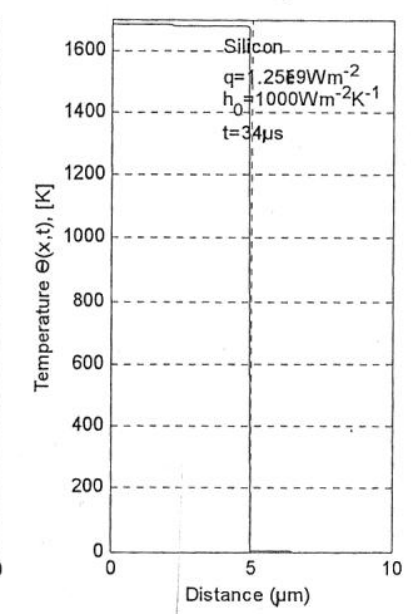

Fig. 3: The variation of the temperature of the thin film of silicon with the distance at $\mathrm{t}=34 \mu \mathrm{s}$. For silicon-glass two layer system. 


\section{International Journal of Science and Research (IJSR) \\ ISSN (Online): 2319-7064}

Index Copernicus Value (2013): 6.14 | Impact Factor (2014): 5.611

\section{Results}

The obtained results reveal that:

1) The critical time required to initiate melting in silicon for the considered operation condition is $t_{m}=34 \mu$ s.

2) The thermal penetration depth in the front Silicon layer after exposure interval of time $\mathrm{t}=34 \mu \mathrm{s}$ for the two layer Silicon - glass system in $5 \mu \mathrm{m}$ for the considered operating conditions.

3) The obtained mathematical expressions for the thermal profiles in the thin film front layer (eq. 40) and that for the substrate layer (eq. 41) reveal clearly that :

a) They depend linearly on the maximum value $q_{\max }$ of the laser source power density.

b) The dependence on the cooling conditions $\left(h_{a}\right.$, $\left.\mathrm{W} / \mathrm{m}^{2} \mathrm{~K}\right)$ is not linear.

c) Such profiles depend on the value of the absorption coefficient at the front surface $\left(\mathrm{A}_{\mathrm{f}}\right)$ of the irradiated system such dependence is linear for $\mathrm{A}_{\mathrm{f}}$ constant.

d)These profiles depend principally on the general shape of the heating laser source pulse, through the parameter "m"

e) The dependence on the pulse duration td and the time "to" required to reach $\mathrm{q}_{\max }$ is revealed through the factor $\left(t_{d}-t_{0}\right)^{m}$. Small values of such factor give higher values for the temperature of the heated target (see equations (40) and (41).

\section{Conclusions}

1) The thermal response of the laser irradiated target is a function of the pulse shape and the operating conditions.

2) The dependence of the temperature field on the maximum power density $\mathrm{q}_{\max }$ of the laser pulse is linear.

3) The dependence of the temperature field within the heated target on the absorption coefficient of constant value is linear.

4) The dependence of the temperature field within the irradiated target on the cooling conditions is not linear.

\section{References}

[1] J.F. Ready, Effects due absorption of laser radiation, J. Appl. Phys. Vol. 36 No. 2, 462-468, 1965.

[2] M.O. Aboelfotoh and R. J. Von Guttfeld, Effects of pulsed laser radiation on thin aluminum films, J. Appl. Phys. Vol. 43 No. 9, 3789 - 3794, 1972.

[3] R.J. Von Gutfeld and Chaudhari, laser writing and erasing on chalcogenide films, J. Appl. Phys. Vol. 43 No. 11, 4688-4693, 1972.

[4] R.A. Ghez and R.A. Loff, laser heating and melting of thin films in low-conductivity substrate, J. Appl. Phys. Vol. 46 No. 5, 2103 - 2110, 1975.

[5] M. Von Allman, laser drilling velocity in metals, J. Appl. Phys. Vol. 47 No. 12, 5460 - 5463, 1976.

[6] E.M. Breinan, B.H. Kear and C.M. Banas, processing materials with lasers, Physics Today, PP. 44-50, (November), 1976.
[7] A. Cutolo, P. Gay and S. Solimeno, Mirrors deformations and wave front aberrations caused by CW high power laser beams, OpticaActa, Vol. 27 No. 8, $1105-1116,1980$.

[8] N. Barakat, T. El-Dessouki, F. Sharaf, Laser Induced Damage to glass and stainless steel targets, J. Optics) Paris), Vol. 11, No. 2, 123 - 126, 1980.

[9] A. Bhattacharyya and B.G. Streetman, Dynamics of pulsed Co2 laser annealing of Silicon, J. Phys. D.: Appl. Phys. Vol. 14 L67 - 72, 1981.

[10] Yu. K. Danileiko, T.P. Lebedeva, A.M. Prokhorov and A.V. Sidorin, Dynamics of nonlinear absorption of light in solids, Sov. Phys. JETP Vol. 57 No. 6, $1183-1187,1983$.

[11] L.D. Merkle, N. Koumvakalis and M. Bass, laser induced bulk damage in $\mathrm{SiO}_{2}$ at 1.064, 0.532 and 0.55 mm, J. Appl. Phys. Vol. 55, No. 3, 772 - 775, 1984.

[12] D. Waechter, P. Schvan, R.F. Thomas and N.G. Tavr, Modeling of heat flow in multilayer $\mathrm{CW}$ laser annealed structures, J. Appl. Phys. Vol. 59 No. 10, $3371-3374,1986$.

[13] A. Abtahi, P.F. Braunlich and P. Kelly, Theory of transient temperature response of a two-layer system heated with localized laser beam, J. Appl. Phys. Vol. 60, No. 10, 3417 - 3421, 1986.

[14] M.K. El-Adawi and E.F. El-Shehawey, Heating a slab induced by a time - dependent laser irradiance an exact solution, J. Appl. Phys. Vol. 60, No. 7, $2250-$ 2255, 1986.

[15] M. K. El-Adawi, laser melting of solids an exact solution for time intervals less or equal to the transit time, J. Appl. Phys. Vol. 60, No. 7, 2256 - 2259, 1986.

[16] M.K. El-Adawi and S.A. Shalaby, laser melting of solids - an exact solution for time intervals solution for time intervals greater than the transit time, J. Appl. Phys. Vol. 60 No. 72260 - 2265, 1986.

[17] M.M. EL-Nicklawy, M.K. El-Adawi, A.A. Kutub, G.G. Al-Barakati, Analytical approach for melting and evaporation of a solid by a pulsed laser, ActaPhysicaHungarica, Vol. 59, 291, 1986.

[18] A. F. Hassan, M.M. El-Nicklawy, M.K. El-Adawi, and A.A. Hemida, a general problem of pulse laser heating of a slab, optics and laser technology, Vol. 25, No. 3, $155-162,1993$.

[19] M.K. El-Adawi, M.A. Abdel-Naby and S.A. Shalaby, laser heating of a two-layer system with constant surface absorption : an exact solution, Int. I heat and mass transfer, Vol. 38 No. 5, 947 - 952, 1995.

[20] A.F. Hassan, M.M. El-Nicklawy, M.K. El-Adawi E.M. Nasr, A.A. Hemida, O.A. Abd El-Ghaffar, Heating effects induced by a pulsed laser in a semiinfinite target in view of the theory of linear systems, Optics \& Laser Technology, Vol. 28, No. 5, 337 , 1996.

[21]Z. H. Shen, S.Y. Zhang, J. Lu, X. W. Ni, Mathematical modeling of laser induced heating and melting in solids, Optics \& Laser Technology, Vol. 33, $533-537,2001$.

[22] M.A. Al-Nimr, M. Alkam, V. Arpaci, heat transfer mechanisms during short - pulse laser heating of two layer composite films, Heat Mars Transfer Vol. 38, $609-614,2002$. 


\section{International Journal of Science and Research (IJSR) \\ ISSN (Online): 2319-7064}

Index Copernicus Value (2013): 6.14 | Impact Factor (2014): 5.611

[23] A. Koc, 3-D analysis of temperature distribution in the material during pulsed laser and material interaction, Heat Mass Transfer, Springer - Verlage 10-1007 No. 1, PP. 1-22, 2003.

[24] M.K. El-Adawi, S.A. Shalaby, S.S. Mostafa, and M.K. Kotkata, laser thermal response of a finite slab as a function of the laser pulse parameters, Optics and Laser Technology 39, PP. 424 - 429, 2007.

[25] M.K. El-Adawi, H,M. Al-Theeb, Heating onedimensional homogeneous slab with pulsed laser in relation to the laser pulse parameters, AJSE, Vol. 33, No. 1. PP. 147 - 162, 2008.

[26] M.K. El-Adawi, H.S. Al-Fanakh, laser heating of a finite silver selenide slab, Journal of Optics \& Laser Technology, Vol. 49, PP. 231 - 236, 2013.

[27] M.K. El-Adawi, I.A. Al-Nuaim, heating of a finite slab with $\mathrm{CW}$ laser in relation to cooling conditions at the rear surface, Material Science Research India, Vol. 10, PP. 17 - 22, 2013.

[28] M.K. El-Adawi, S.A. Shalaby, S.E.S. Abdul Ghany, Interaction of laser radiation with solids Review Articles, International Journal of Natural Sciences, Research (IJNSR), 82, 2015.

[29]E.D. Rainville and P.E. Bedient, Elementary Differential Equations, McMillan, $5^{\text {th }}$ edition, Ch. 25, P. 465, New York, 1974.

[30]F. Oberhettinger and L. Badii, Tables of Laplace Transforms, Springer - Verlag, New York, 1973.

[31] M.R. Spiegel, Laplace Transforms, Schaum's Outline Series, McGraw-Hill, New York, 1965.

[32] E.R.G. Eckert and R.M. Drake, Analysis of Heat and Mas Transfer, Int. student edition, McGraw-Hill, Kogakusha, Ltd. Japan, 1972.

[33] S.M. Sze, Physics of semiconductors devices, JohnWiley \& Sons, New York, 1981.

[34]R.C. Weast Handbook of Chemistry and Physics $159^{\text {th }}$ edition CRC Press, Florida, 1979.

\section{NOMECLATURE}

$A_{f}$ Optical surface absorptance of the thin film front layer, dimensionless.

$\mathrm{C}_{\mathrm{p}}$ Specific heat $\left[\mathrm{J} \mathrm{kg}^{-1} \mathrm{~K}^{-1}\right]$

$\mathrm{d}$ Thickness of the thin film [m].

$\mathrm{h}_{\mathrm{o}}$ Heat transfer coefficient at the front surface $\left[\mathrm{Wm}^{-2} \mathrm{~K}^{-1}\right]$.

$\mathrm{m}$ A fitting parameter defined in the text, dimensionless.

$\mathrm{q}_{\mathrm{o}}$ Laser irradiance $\left[\mathrm{Wm}^{-2}\right]$.

S Laplace transform variable that stands for the time variable t.

$\mathrm{T}$ The temperature in degrees Kelvins

$\mathrm{T}_{\mathrm{m}}$ The melting temperature $[\mathrm{K}]$.

t Time variable $[\mathrm{s}]$

$\mathrm{t}_{\mathrm{m}}$ Time interval required to initiate damage [s].

$\mathrm{t}_{\mathrm{o}}$ The time interval required to reach $\mathrm{q}_{\max }$.

$\mathrm{x}, \mathrm{z}$ Spatial variables $[\mathrm{m}]$.

\section{Greek Symbols:}

$\alpha$ Thermal diffusivity $\left[\mathrm{m}^{2} \mathrm{~S}^{-1}\right]$

$\lambda$ Thermal conductivity $\left[\mathrm{Wm}^{-1} \mathrm{~K}^{-1}\right]$

$\rho$ Density of the material $\left[\mathrm{kg} \mathrm{m}^{-3}\right]$

$\theta$ Excess temperature relative to the ambient temperature $\left(\mathrm{T}-\mathrm{T}_{0}\right) \cdot[\mathrm{K}]$.

\section{Subscripts:}

f Related to the film layer. $\mathrm{p}$ related to the substrate layer 